\title{
Significance of calorie-restricted ketogenic diet for lung cancer with brain metastases and hepatoma with pulmonary metastases: report of two cases
}

Dong TAN ( $\square$ tandongcn@126.com )

Shandong University Qilu Hospital https://orcid.org/0000-0001-5921-0393

Jing Zhao

Cambridge Stem Cell Institute

Xiao-jing Liu

Jinan Municipal Hospital

Wen-ru Yang

Shandong University Qilu Hospital

Fang Yuan

Shandong University Qilu Hospital

Brief communication

Keywords: Cancer, metastases, Warburg effect, calorie restriction, ketogenic diet

Posted Date: April 9th, 2020

DOI: https://doi.org/10.21203/rs.3.rs-21217/v1

License: (9) This work is licensed under a Creative Commons Attribution 4.0 International License.

Read Full License 


\section{Abstract}

Cancer cells have altered metabolism that is characterized by an enhanced uptake and utilization of glucose. These increased glucose dependence alterations, present potential vulnerabilities that could be targeted for cancer therapy. The calorie-restricted ketogenic diet (KD) may meet the requirement. In this study, we explore the feasibility of the ketogenic diet as adjuvant therapy for cancer treatment. We present two cases of patients diagnosed with aggressive forms of cancer, lung cancer with brain metastatic tomours and primary liver cancer with pulmonary metastases. The patients began KD intervention after exhausting the other treatment options. Both of them responded to KD and demonstrated a beneficial effect. The tomour size from serial imaging and serum tumour markers were significantly reduced after KD in both subjects without any adverse effects. Both patients had tumour remission even after stopping the ketogenic diet, indicating that KD is a safe, effective treatment to aggressive cancers with metastases when used with conventional therapies.

\section{Introduction}

The metabolism of cancer cells differs significantly from that of normal cells and these differences play an important role in tumorigenesis [1]. In cancer cells, most energy comes from the conversion of glucose to lactate, even in the presence of normal levels of oxygen. This metabolic shift from oxidative phosphorylation to glycolysis is known as the Warburg effect [1]. The Warburg effect exposes fundamental vulnerabilities of cancer cells reliance on excess glucose for survival and proliferation. Under the glucose deprivation condition, cancer cells are not capable to switch to ketone bodies as a substitute for energy, due to mitochondrial dysfunction and down-regulation of enzymes necessary for ketone utilization [2]. Lacking the metabolic flexibility of cancer cells presents a therapeutic potential for cancer treatment.

The Ketogenic Diet (KD), a high-fat/low-carbohydrate/adequate-protein diet that originally used to treat epilepsy $[3,4]$, has recently been suggested as adjuvant therapy in cancer treatment $[5,6]$. KD aims at the Warburg effect, causing cancer cell death by glucose starvation while normal cells adapt their metabolism to utilize ketone bodies as a substitute for energy, on which normal cells survive. Furthermore reducing blood glucose is also associated with reduced levels of insulin and insulin-like growth factor, which play an important role in cancer cell proliferation [7]. Numerous preclinical studies have provided evidence for an anti-tomour effect of KD [5, 6, 8-12]. The clinical potential of KD in the treatment of cancer has only recently been explored. Most of the clinical data are from case reports [13-17] or pilot studies mainly focusing on the safety of the $\operatorname{KD}[18,19]$. Only one randomized controlled trial is available to date [20]. The results of these clinical trials were encouraging, specifically for brain tumour $[5,13,14]$. Importantly no serious adverse effect or toxicity related to KD intervention in these studies. We present here two case studies of patients diagnosed with lung cancer with intracranial metastatic tumours and primary liver cancer with pulmonary metastases. Both patients started KD after failed conventional treatments. Our study confirmed the beneficial effect of KD in cancer treatment. 


\section{Case Report}

\section{Case one}

A 32 years old female who was six months pregnant had experienced cough without obvious inducement in 2012. The cough was dry and not accompanied by sputum, chest tightness and wheezing or fever. At that time, the patient did not seek any medical advice, diagnosis or treatment. The cough was relieved after giving birth. A few months after giving birth, the patient began to experience cough symptoms again with white phlegm, however, no diagnosis or treatment was given. After that, the patient's cough was aggravated, accompanied by chest tightness and asthma. Chest CT examination in the affiliated hospital of Qingdao University showed multiple plaques and consolidation in both lungs where fluid dark areas were visible and a large amount of pleural effusion was on the left side. No swelling of mediastinal lymph nodes was observed. Laboratory test results showed that carcinoembryonic antigen (CEA) at $50.05 \mathrm{ng} / \mathrm{ml}$, neuron-specific enolase (NSE) $18.96 \mathrm{ng} / \mathrm{ml}$, cancer antigen (CA) $12561.67 \mathrm{u} / \mathrm{ml}$ and erythrocyte sedimentation rate $67 \mathrm{~mm} / \mathrm{h}$. Closed thoracic drainage was given and fluid drainage had a reddish-brown appearance. About a total of $1000 \mathrm{ml}$ of fluid was drained out. Concurrent puncture biopsy confirmed invasive adenocarcinoma. The patient was then admitted to our hospital.

Chest CT images collected in 19th November 2014 showed multiple and high lung density, which was consistent with the previous CT findings of lung cancer, as well as left pleural effusion and left inferior lobe atelectasis (Fig. 1). Lung biopsy confirmed invasive adenocarcinoma, and laboratory test results showed elevated tumour markers: CEA $16.08 \mathrm{ng} / \mathrm{ml}$; carbohydrate antigen 125 (CA-125) $63.80 \mathrm{U} / \mathrm{ml}$; cancer antigen $15-3$ (CA15-3) $79.62 \mathrm{U} / \mathrm{ml}$. During the hospitalization, the patient's condition gradually worsened, with continuous intermittent fever, accompanied by repeated chest tightness and suffocation. Despite anti-infection therapy was given, the patient's condition was still progressive aggravation. A panel of immunohistochemistry staining showed: TTF-1 (+), CK7(+), Syn(-), CgA(-), Ki67 positive rate was about $5 \%$, EML4-ALK variant 1 (gene fusion point E13; A20) and variant 3a/b (gene fusion point E6aE; A20) were positive. Crizotinib (200 mg bid) was given on 13th December 2014, and the patient's symptoms gradually improved.

The patient was readmitted one year later after complaining of headache and nausea on $24^{\text {rth }}$ February 2016. Brain MRI scan obtained on 26th February 2016 revealed multiple lesions, indicating brain metastases (Fig. 2). DWI and FLAIR images showed multiple metastatic lesions with peripheral edema with the brain parenchyma.

The patient was instructed to a strict KD together with continued Crizotinib treatment on 3rd March 2016. No apparent ketosis or significantly decreased blood sugar was observed during KD interventions. Although the patient only had the strict KD for two and a half months due to feeling unable to tolerant the strict diet, the clinical symptoms and signs were remarkably improved. Both the chest CT scan and brain MRI scan showed significant changes. All the previous lesions in the lungs (Fig. 3A-C) and brain (Fig. 4) were deceased gradually by days. Pulmonary lesions were almost complete resolution two months after 
KD on 3rd May 2016 and showed no recurrent tumour in follow-up chest scan on 8th May 2017 (Fig. 3AC). Brain metastatic lesions were almost undetectable six months after KD and complete resolution in subsequent brain MRI scans performed on 29th September 2016 and 8th May 2017 (Fig. 4). The lasted chest CT and brain MRI performed in December 2019 did not show any abnormal nodules (data not shown), indicating the patient had remission nearly 4 years after treatments.

Consistent with the tumour volume, the levels of serum tumour markers declined gradually and finally fell within the normal range with CEA $2.2 \mathrm{ng} / \mathrm{ml}$, CA-125 25.41 and CA15-3 16.92 in 18th December 2016) (Fig. 3D-F).

\section{Case Two}

A 47 years old female patient had a history of hepatitis B for 23 years and was admitted to the hospital for 6 days due to right upper abdominal pain. On 15th July 2018, a screening test showed that the levels of alpha fetoprotein (AFP) level in the blood were elevated, AFP $\geq 2000 \mathrm{ng} / \mathrm{ml}$, ferritin $210.24 \mathrm{ng} / \mathrm{ml}$, and neuron-specific enolase $25.20 \mathrm{ng} / \mathrm{ml}$. Chest CT showed double pulmonary fibre foci. Abnormal intrahepatic density was also observed. Two days later, enhanced CT scan was performed to confirm the lesion of liver mass, which was consistent with the manifestations of liver cancer. The left lobe of the liver was removed on 21st July 2018. Liver pathological examination results showed grade II hepatocellular carcinoma and nodular cirrhosis. The patient recovered well after surgery and was discharged 10 days later. No special follow-up treatment was administered.

After discharge from the hospital, the patient continuously monitored AFP index in the blood and found that there was a constant increase. AFP level reached $5713 \mathrm{ng} / \mathrm{ml}$ on $21 \mathrm{st}$ December 2018. CT scan revealed multiple enhanced nodules in the abdominal liver, suggesting tumour recurrence. Multiple nodular lesions were detected in the middle and lower lobes of the lung on 20th November 2018 (Fig. 5AD), indicating pulmonary metastases.

Considering the aggressive nature of cancer and limited treatment options available, the patient was instructed to start a ketogenic diet on 21st December 2018. The proportion of fat in the dietary was approximate $30 \%$; the proportion of protein was approximately $50-60 \%$; the proportion of vegetable and cellulose was approximately $10-20 \%$. The patient came off KD after one month since she found it difficult to maintain the strict KD. No apparent ketosis or significantly decreased blood sugar was observed during KD interventions. After one month of strict KD, AFP levels reduced to $512 \mathrm{ng} / \mathrm{ml}$. AFP levels fell within a normal range at $3.01 \mathrm{ng} / \mathrm{ml}$ two months later and maintained AFP levels $1.41 \mathrm{ng} / \mathrm{ml}$ half a year later (Fig. 6). Consistent with this, the CT scan revealed much reduced pulmonary nodules $d$ in the middle lobes of the lung and no residual nodules detected in the lower lobes of the lung on 24th July 2019 (Fig. 5E-H). The latest scan images collected on 20th December 2019 showed no recurrent tomour (data not shown), indicating that the tumour in complete remission one and a half years after KD.

\section{Discussion}


Cancer metabolism is an essential aspect of tumorigenesis and is one of the oldest research areas in cancer biology [21]. Despite a broad range of metabolic alterations has been reported in tumours recently the Warburg effect is still the most recognized metabolic characteristic of many cancer cells [1]. Tumour proliferation and survival are dependent upon these metabolic changes to provide the excess needs for glucose [1]. The altered metabolism in cancer cells, therefore, provided a unique therapeutic target to treat cancer. This study explored the feasibility of calorie-restricted KD to target the Warburg effect in the treatment of patients with cancer.

Nutrient sensing is essential for cell survival and a regulator for cell function. Cellular homeostasis is maintained by sensing changes in nutrient availability, followed by adaptive responses [22]. While normal cells metabolise glucose for energy under normal physiological conditions, they can utilise ketone bodies a substitute for glucose when blood glucose levels decrease [23, 24]. The dynamic metabolic adaptation is particularly critical for brain function and cognition. Ketone bodies can cross the blood-brain barrier and play a vital role as energy substrates for the brain, especially during times of starvation and in early childhood brain development [25-27]. Ketones can provide as much as $70 \%$ of the brain's energy needs, more efficiently than glucose [28]. Tumour cells, however, lack the dynamic metabolic adaptation. The result is that the cancer cells become heavily dependent on glucose and are unable to utilize the ketones as a substitute for glucose. It has been shown that higher glucose could enhance cancer aggressiveness [29]. A "vicious cycle" may exist within the cancer cells which is that glucose drives cancer progress whilst metabolic changes in cancer may favour glucose delivery to cancer cells [30]. Restriction of glucose will break this vicious cycle thereby inhibit tumour progression.

Two patients with aggressive forms of cancers were recruited for our study, a 32-year-old lung cancer patient with brain metastases, and a 47-year-old liver cancer patient with pulmonary metastases. Both patients had poor prognoses. A strict KD was administered to them after they had exhausted the conventional anti-tumour treatment options. They demonstrated excellent therapeutic responses to KD. Both patients had improved clinical symptoms and the tumour size reduced progressively without adversely affecting the patient's overall nutritional status. Although patients were only on KD for two and a half months for the patient with brain metastases in lung cancer, and one month for the patient with pulmonary metastases in liver cancer, the anti-tomour effect of KD was evident and long-lasting. The recent physical exams and scan images revealed that the cancers had gone into remission in both patients despite both of patients discontinuing the KD. This profound and lasting beneficial effect of KD may be due to the following reasons: 1) KD targets the Warburg effect, limits the glucose availability and selectively starves tomours. It is particularly effective for the aggressive forms of cancers where excess glucose is required to support tumour proliferation and progression. 2) After initial reducing the tumour size and slowing the tumour growth rate, the immune system could match the rate of tumour proliferation, regain control over tomour growth and establish a new stage of equilibrium. 3) It has been reported that KD could act as an immune adjuvant, increase tomour-reactive innate and adaptive immune responses [31]. 4) KD may not only be responsible for the restriction of nutrient uptake in tomour cells, but Ketone bodies may also influence the epigenetic regulation of gene expression, involving several possible downstream pathway interactions to inhibit the tumour growth and proliferation. Indeed, the 
previous studies showed that KDs could influence the patterns of gene expression in tomours [32, 33].

The long-lasting beneficial effect of KD on cancer patients suggests a synergistic action of KD.

\section{Conclusion}

We have demonstrated the significant beneficial effect of a calorie-restricted ketogenic diet for two types of aggressive cancers with metastases. Even a brief KD intervention has a profound effect, implicating the significant therapeutic potential for KD, especially for the fast-growing and aggressive forms of cancers. Compared to other anti-cancer drugs and standard treatment, KD is safe, effective, inexpensive and easy to implement. With recently much improved KD recipes, KD may warrant routine application as adjuvant therapy in cancer treatment in future.

\section{Abbreviations}

KD Ketogenic diet

CT Computerised tomography

MRI Magnetic resonance imaging

DWI Diffusion weighted imaging

FLAIR Fluid-attenuated inversion recovery

CEA Carcinoembryonic antigen

NSE Neuron-Specific Enolase

CA Carbohydrate antigen

TTF-1 Thyroid transcription factor 1

CK7 Cytokeratin 7

Syn Synaptophysin

CgA Chromogranin A

Ki67 Antigen KI-67

EML4 Echinoderm microtubule-associated protein-like 4

ALK Anaplastic lymphoma kinase

AFP Alpha fetoprotein 


\section{Declarations}

Ethics Declarations

Written informed consent was provided by the study participants, and the study protocol was approved by the Ethics Committee of the Qilu Hospital of Shandong University (Qingdao).

Consent For Publication

Not applicable.

Availability Of Data And Materials

The data used in this study are available from the corresponding author on request.

Competing interests

The authors declare that they have no competing interests.

\section{Funding}

This work was supported by Department of General Practitioner, Qilu Hospital of Shandong University (Qingdao).

Authors' contributions

DT carried out designed the study, responsible for the patient management, analysis the data and drafted the manuscript. JZ helped reviewed and revised the manuscript and contributed to the discussion. XJL, WRY and FY participated in the patient management and clinical data collection. All authors read and approved the final manuscript.

Acknowledgements

We thank all the participants for their contributions to this study.

\section{References}

1. Weyandt JD, Thompson CB, Giaccia AJ, Rathmell WK. Metabolic Alterations in Cancer and Their Potential as Therapeutic Targets. Am Soc Clin Oncol Educ Book. 2017;37:825-32.

2. Klement RJ, Kammerer U. Is there a role for carbohydrate restriction in the treatment and prevention of cancer? Nutr Metab (Lond). 2011;8:75.

3. Nei M, Ngo L, Sirven JI, Sperling MR. Ketogenic diet in adolescents and adults with epilepsy. Seizure. 2014;23:439-42.

4. Li HF, Zou Y, Ding G. Therapeutic Success of the Ketogenic Diet as a Treatment Option for Epilepsy: a Meta-analysis. Iran J Pediatr. 2013;23:613-20.

5. Klement RJ. Fasting, Fats, and Physics: Combining Ketogenic and Radiation Therapy against Cancer. Complement Med Res. 2017;25:102-13.

6. Weber DD, Aminzadeh-Gohari S, Tulipan J, Catalano L, Feichtinger RG, Kofler B: Ketogenic diet in the treatment of cancer - Where do we stand? Mol Metab 2019. 
7. Brahmkhatri VP, Prasanna C, Atreya HS. Insulin-like growth factor system in cancer: novel targeted therapies. Biomed Res Int. 2015;2015:538019.

8. Morscher RJ, Aminzadeh-Gohari S, Hauser-Kronberger C, Feichtinger RG, Sperl W, Kofler B. Combination of metronomic cyclophosphamide and dietary intervention inhibits neuroblastoma growth in a CD1-nu mouse model. Oncotarget. 2016;7:17060-73.

9. Shukla SK, Gebregiworgis T, Purohit V, Chaika NV, Gunda V, Radhakrishnan P, Mehla K, Pipinos II, Powers R, Yu F, Singh PK. Metabolic reprogramming induced by ketone bodies diminishes pancreatic cancer cachexia. Cancer Metab. 2014;2:18.

10. Rieger J, Bahr O, Maurer GD, Hattingen E, Franz K, Brucker D, Walenta S, Kammerer U, Coy JF, Weller M, Steinbach JP. ERGO: a pilot study of ketogenic diet in recurrent glioblastoma. Int J Oncol. 2014;44:1843-52.

11. Tisdale MJ, Brennan RA, Fearon KC. Reduction of weight loss and tumour size in a cachexia model by a high fat diet. Br J Cancer. 1987;56:39-43.

12. Morscher RJ, Aminzadeh-Gohari S, Feichtinger RG, Mayr JA, Lang R, Neureiter D, Sperl W, Kofler B. Inhibition of Neuroblastoma Tumor Growth by Ketogenic Diet and/or Calorie Restriction in a CD1-Nu Mouse Model. PLoS One. 2015;10:e0129802.

13. Zuccoli G, Marcello N, Pisanello A, Servadei F, Vaccaro S, Mukherjee P, Seyfried TN. Metabolic management of glioblastoma multiforme using standard therapy together with a restricted ketogenic diet: Case Report. Nutr Metab (Lond). 2010;7:33.

14. Strowd RE, Cervenka MC, Henry BJ, Kossoff EH, Hartman AL, Blakeley JO. Glycemic modulation in neuro-oncology: experience and future directions using a modified Atkins diet for high-grade brain tumors. Neurooncol Pract. 2015;2:127-36.

15. Iyikesici MS, Slocum AK, Slocum A, Berkarda FB, Kalamian M, Seyfried TN. Efficacy of Metabolically Supported Chemotherapy Combined with Ketogenic Diet, Hyperthermia, and Hyperbaric Oxygen Therapy for Stage IV Triple-Negative Breast Cancer. Cureus. 2017;9:e1445.

16. Branca JJ, Pacini S, Ruggiero M. Effects of Pre-surgical Vitamin D Supplementation and Ketogenic Diet in a Patient with Recurrent Breast Cancer. Anticancer Res. 2015;35:5525-32.

17. Schwartz K, Chang HT, Nikolai M, Pernicone J, Rhee S, Olson K, Kurniali PC, Hord NG, Noel M. Treatment of glioma patients with ketogenic diets: report of two cases treated with an IRB-approved energy-restricted ketogenic diet protocol and review of the literature. Cancer Metab. 2015;3:3.

18. Iyikesici MS. Feasibility study of metabolically supported chemotherapy with weekly carboplatin/paclitaxel combined with ketogenic diet, hyperthermia and hyperbaric oxygen therapy in metastatic non-small cell lung cancer. Int J Hyperthermia. 2019;36:446-55.

19. Tan-Shalaby JL, Carrick J, Edinger K, Genovese D, Liman AD, Passero VA, Shah RB. Modified Atkins diet in advanced malignancies - final results of a safety and feasibility trial within the Veterans Affairs Pittsburgh Healthcare System. Nutr Metab (Lond). 2016;13:52.

20. Cohen CW, Fontaine KR, Arend RC, Soleymani T, Gower BA. Favorable Effects of a Ketogenic Diet on Physical Function, Perceived Energy, and Food Cravings in Women with Ovarian or Endometrial 
Cancer: A Randomized, Controlled Trial. Nutrients 2018;10.

21. Pavlova NN, Thompson CB. The Emerging Hallmarks of Cancer Metabolism. Cell Metab. 2016;23:27-47.

22. Hietakangas V, Cohen SM. Regulation of tissue growth through nutrient sensing. Annu Rev Genet. 2009;43:389-410.

23. Cunnane SC, Courchesne-Loyer A, St-Pierre V, Vandenberghe C, Pierotti T, Fortier M, Croteau E, Castellano CA. Can ketones compensate for deteriorating brain glucose uptake during aging? Implications for the risk and treatment of Alzheimer's disease. Ann N Y Acad Sci. 2016;1367:12-20.

24. Hasselbalch SG, Knudsen GM, Jakobsen J, Hageman LP, Holm S, Paulson OB. Blood-brain barrier permeability of glucose and ketone bodies during short-term starvation in humans. Am J Physiol. 1995;268:E1161-6.

25. Nehlig A. Brain uptake and metabolism of ketone bodies in animal models. Prostaglandins Leukot Essent Fatty Acids. 2004;70:265-75.

26. Prins ML. Cerebral ketone metabolism during development and injury. Epilepsy Res. 2012;100:21823.

27. Wyss MT, Jolivet R, Buck A, Magistretti PJ, Weber B. In vivo evidence for lactate as a neuronal energy source. J Neurosci. 2011;31:7477-85.

28. White H, Venkatesh B. Clinical review: ketones and brain injury. Crit Care. 2011;15:219.

29. Santos JM, Hussain F. Higher Glucose Enhances Breast Cancer Cell Aggressiveness. Nutr Cancer 2019:1-13.

30. Schwartsburd P. Cancer-Induced Reprogramming of Host Glucose Metabolism: "Vicious Cycle" Supporting Cancer Progression. Front Oncol. 2019;9:218.

31. Lussier DM, Woolf EC, Johnson JL, Brooks KS, Blattman JN, Scheck AC. Enhanced immunity in a mouse model of malignant glioma is mediated by a therapeutic ketogenic diet. BMC Cancer. 2016;16:310.

32. Stafford P, Abdelwahab MG, Kim DY, Preul MC, Rho JM, Scheck AC. The ketogenic diet reverses gene expression patterns and reduces reactive oxygen species levels when used as an adjuvant therapy for glioma. Nutr Metab (Lond). 2010;7:74.

33. Scheck AC, Abdelwahab MG, Fenton KE, Stafford P. The ketogenic diet for the treatment of glioma: insights from genetic profiling. Epilepsy Res. 2012;100:327-37.

\section{Figures}



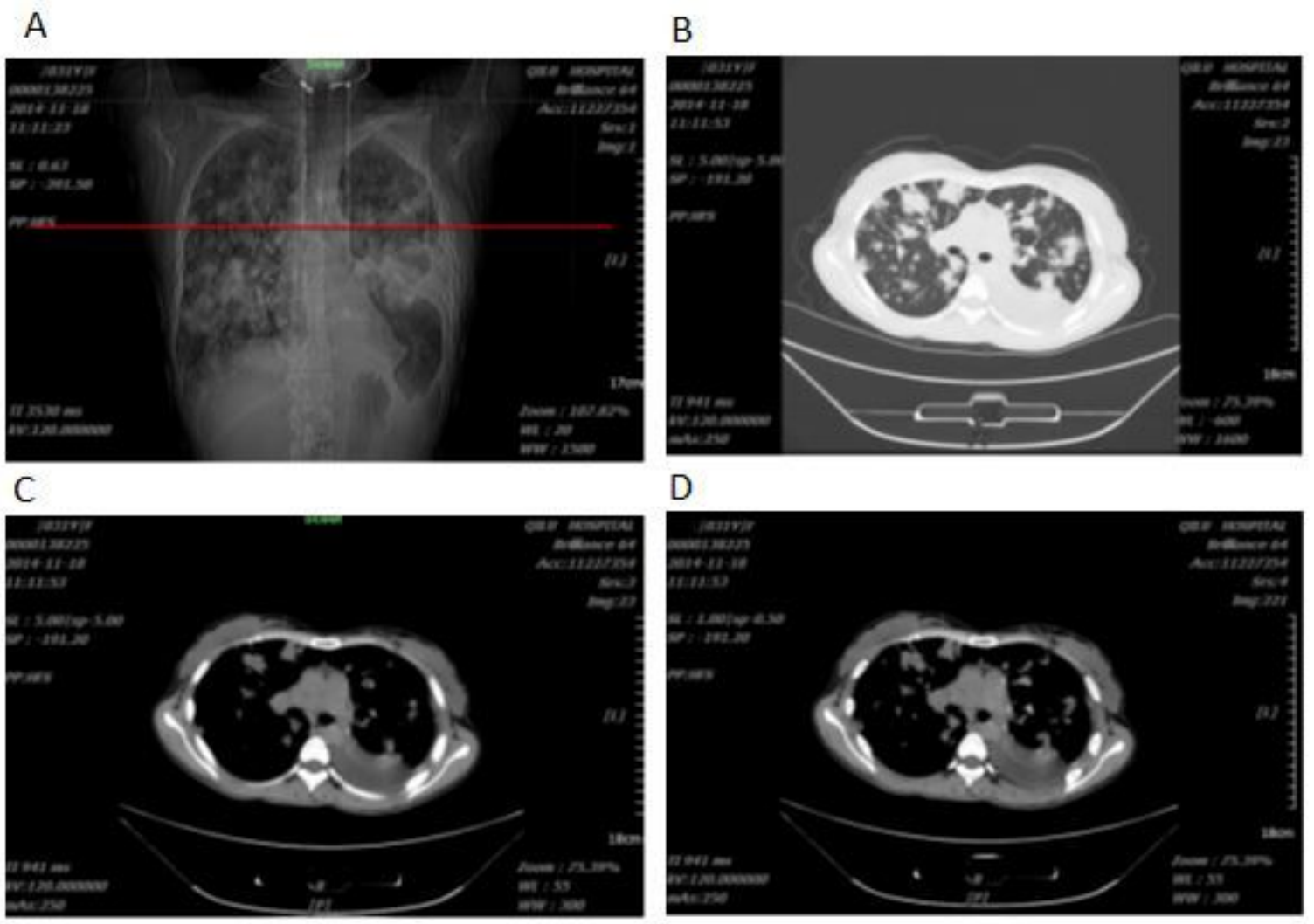

\section{Figure 1}

Chest CT scan from the female patient in Case 1 on 19th November 2014. Suspected lung cancer with bilateral multifocal consolidations revealed by an initial anterior-posterior chest radiograph (A), CT scan in lung window (B) and CT scan in a soft-tissue window (C, D). 
A

B
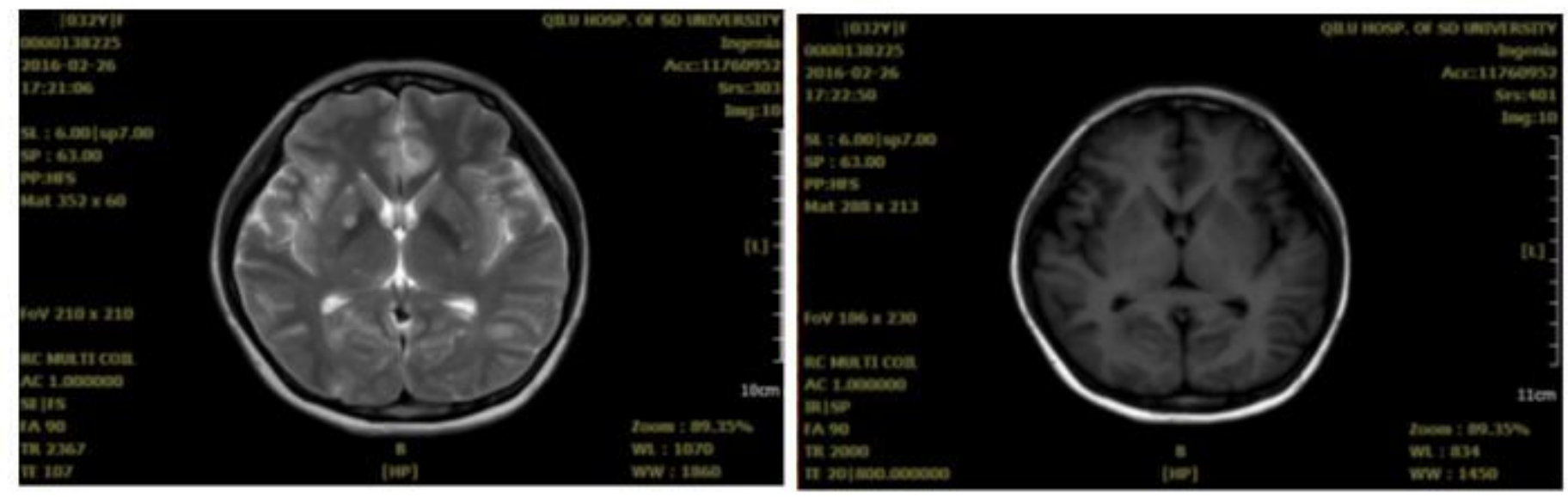

$\mathrm{C}$

D
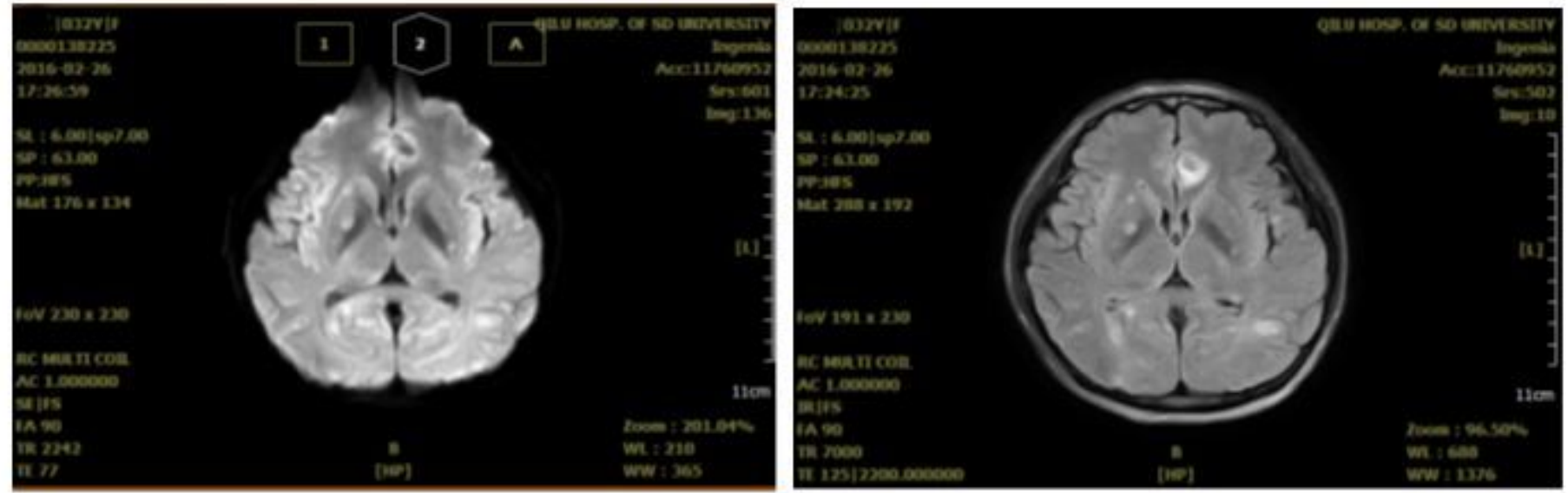

Figure 2

Brain MRI from the female patient in Case 1 on 26th February 2016. The T2-weighted MRI (A) and T1weighted MRI (B) reveal multiple well-defined lesions. DWI (C) shows the metastatic lesions with peripheral edema within the brain parenchyma and FLAIR image (D) shows multiple enhancing metastatic lesions. 
A

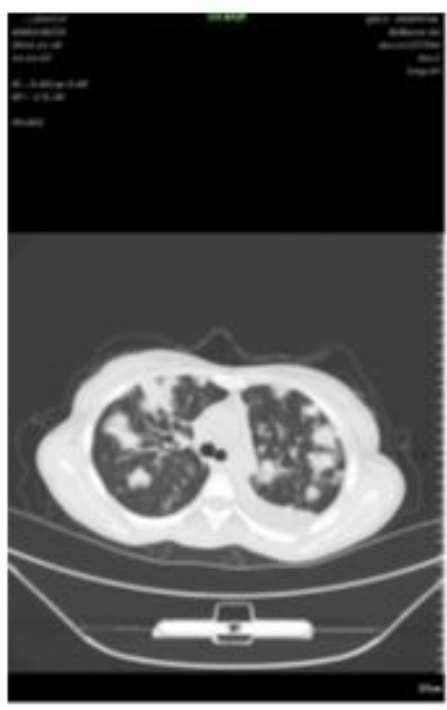

B

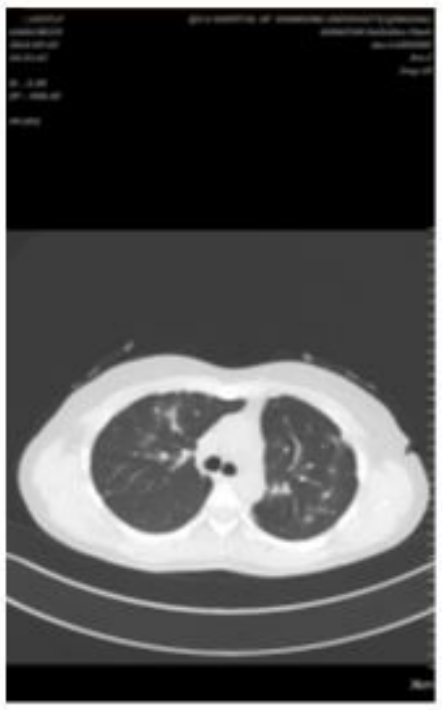

E

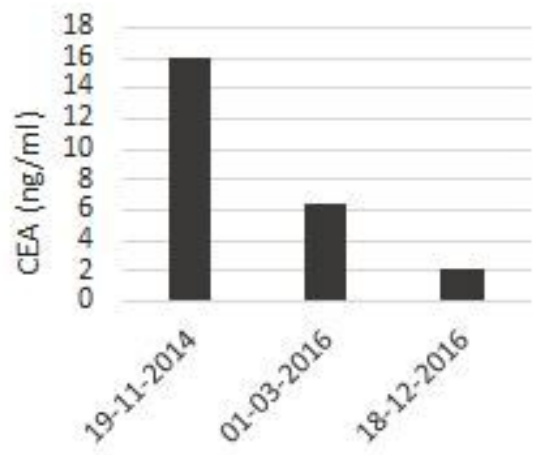

Sampling date

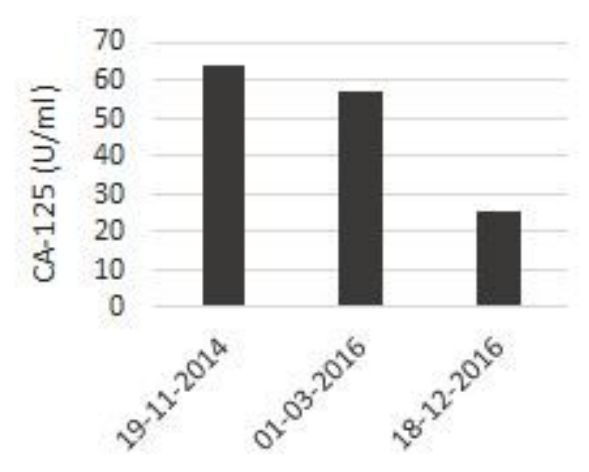

Sampling date

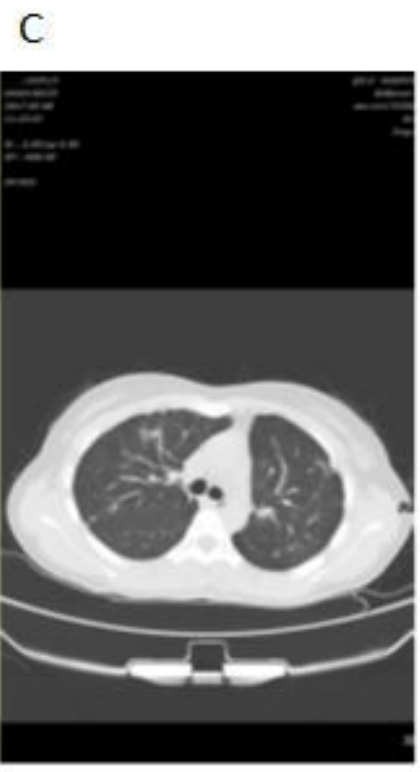

$\mathrm{F}$

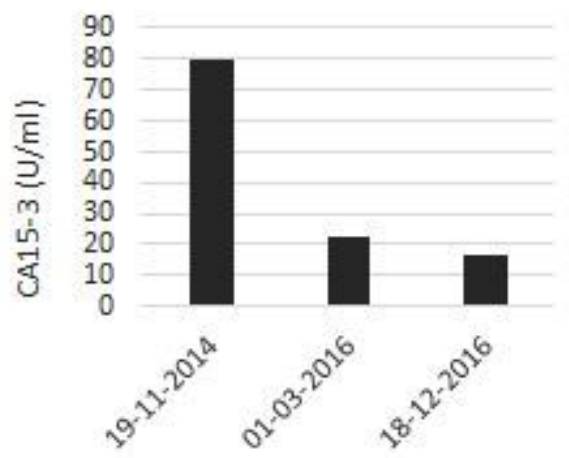

Sampling date

\section{Figure 3}

Changes in the tumour volume and tumour markers of the patient 1 after KD intervention. Chest CT scans revealed multifocal consolidations with ground-glass opacity before KD on 18th November 2014 (A); almost complete resolution of the lesions one and half years after KD on 3rd May 2016 (B); complete remission two and half years after KD on 8th May 2017 (C). The serum levels of carcinoembryonic antigen (CEA) (D), carbohydrate antigen 125 (CA-125) (E) and carcinoembryonic antigen 15-3 (CA 15-3) (F) changed over time and decreased after KD intervention. 
A

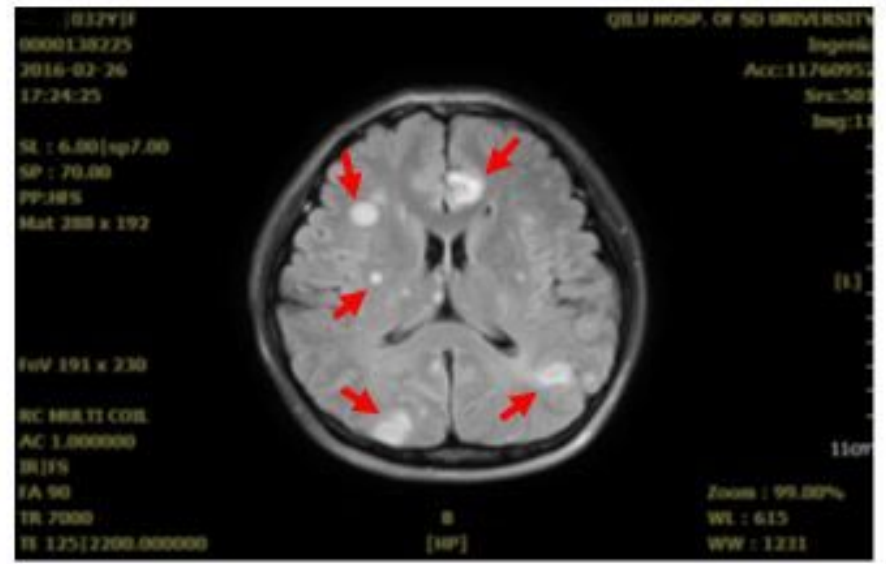

C

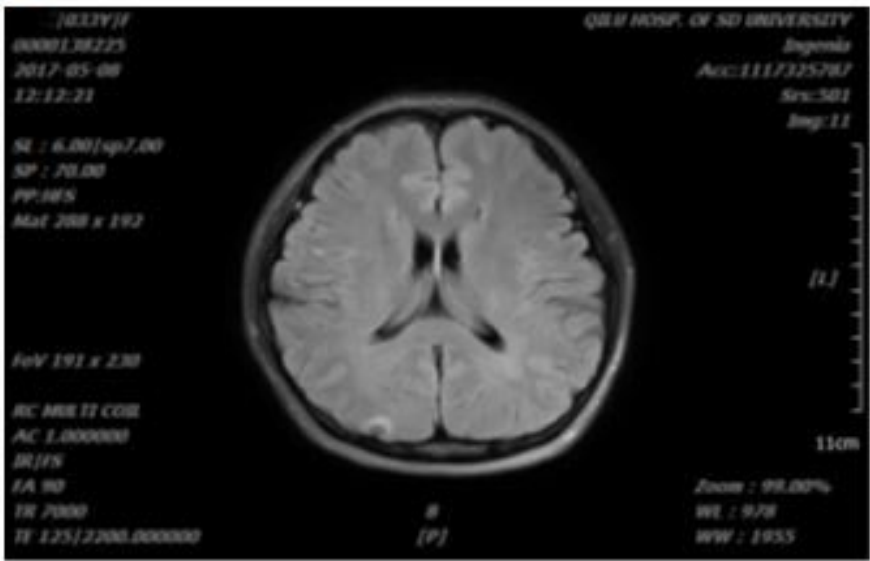

B

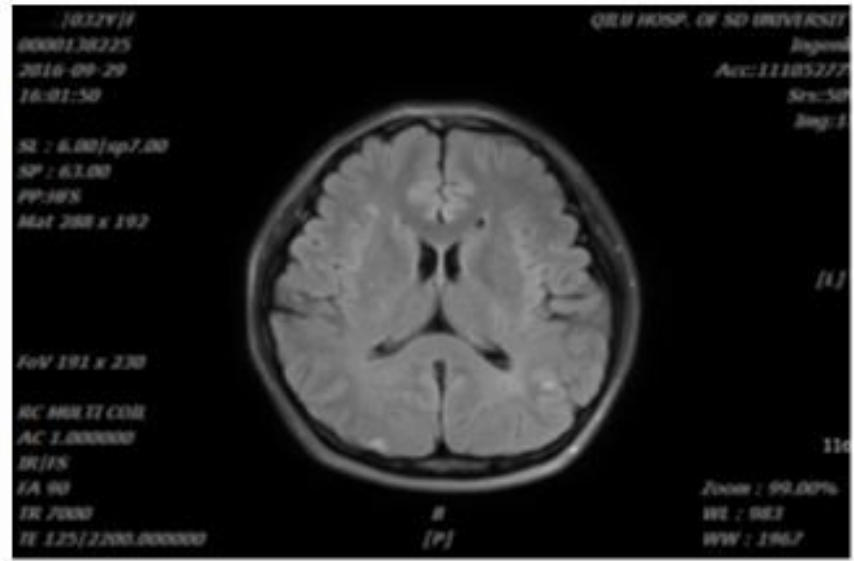

D

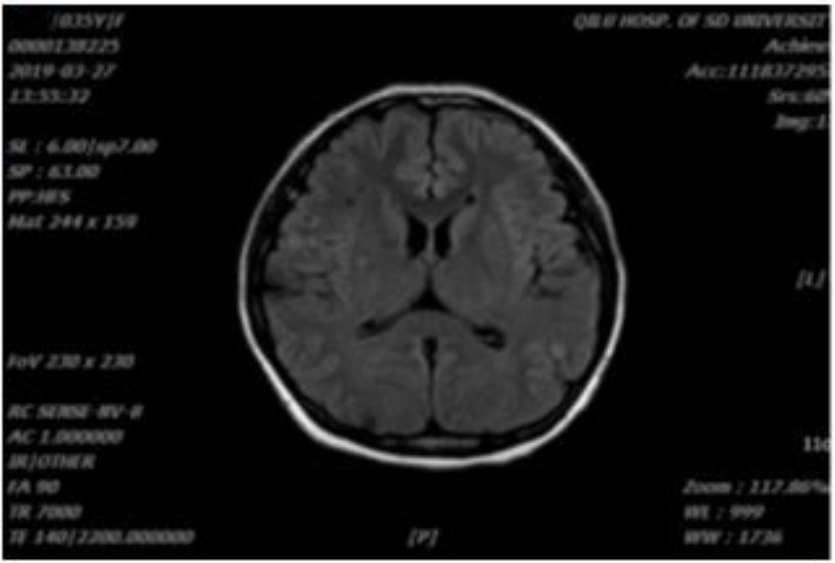

\section{Figure 4}

Brain MRI from the female patient in Case 1. FLAIR images show several metastatic lesions (red arrows) on 26th February 2016 (A) before KD intervention; almost complete resolution of the lesions two months after KD on 29th September 2016 (B); completely remission after KD on 8th May 2017 (C) and on 27th March 2019 (D). 
A

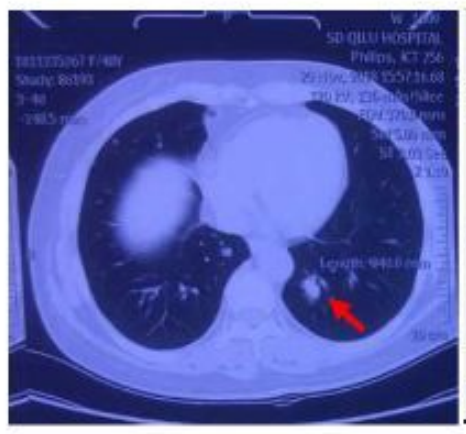

$\mathrm{E}$

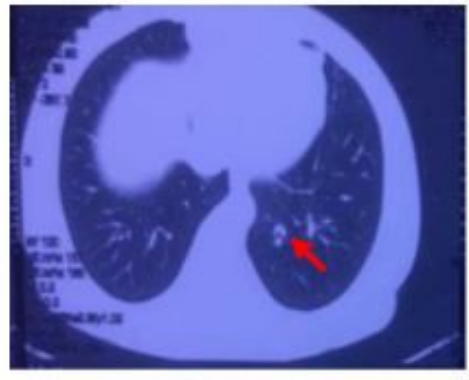

B

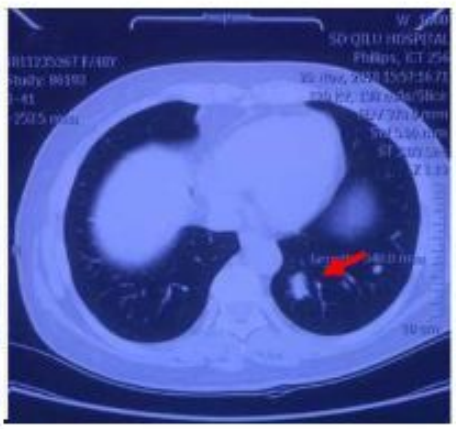

$\mathrm{F}$

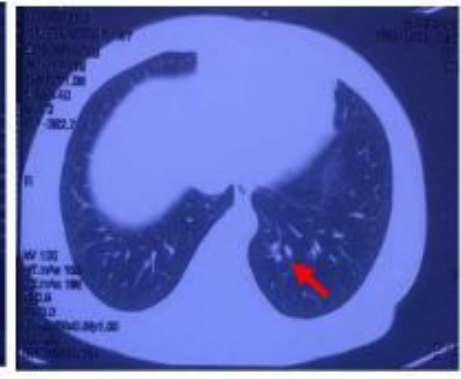

$\mathrm{C}$

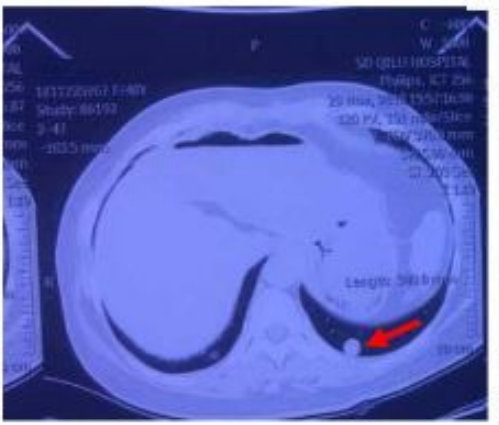

G

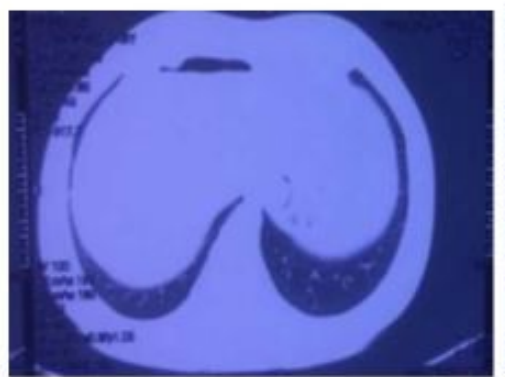

D

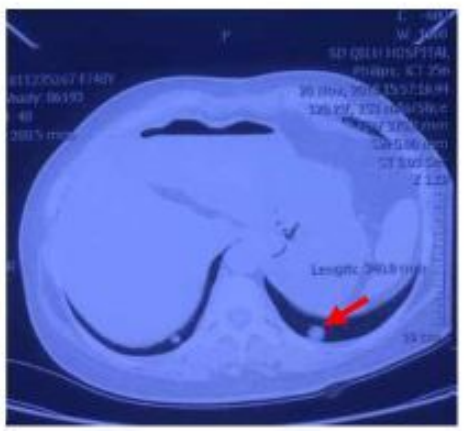

$\mathrm{H}$

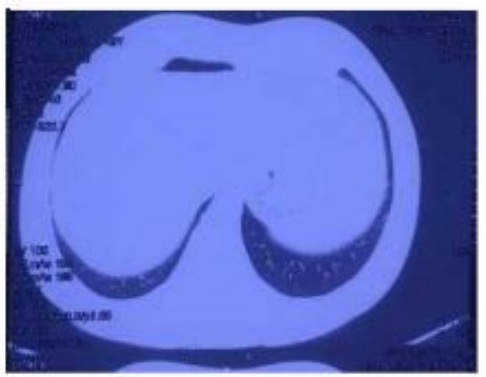

Figure 5

Chest CT scans in lung window of the female patient in Case 2. Multiple pulmonary nodules (red arrows), due to metastases, detected in the middle $(A, B)$ and lower $(C, D)$ lobes of the lung on 20th November 2018. One year after KD intervention. much reduced pulmonary nodules observed in the middle $(E, F)$ and no pulmonary nodules detected in the lower $(G, H)$ lobes of the lung on 24th July 2019.

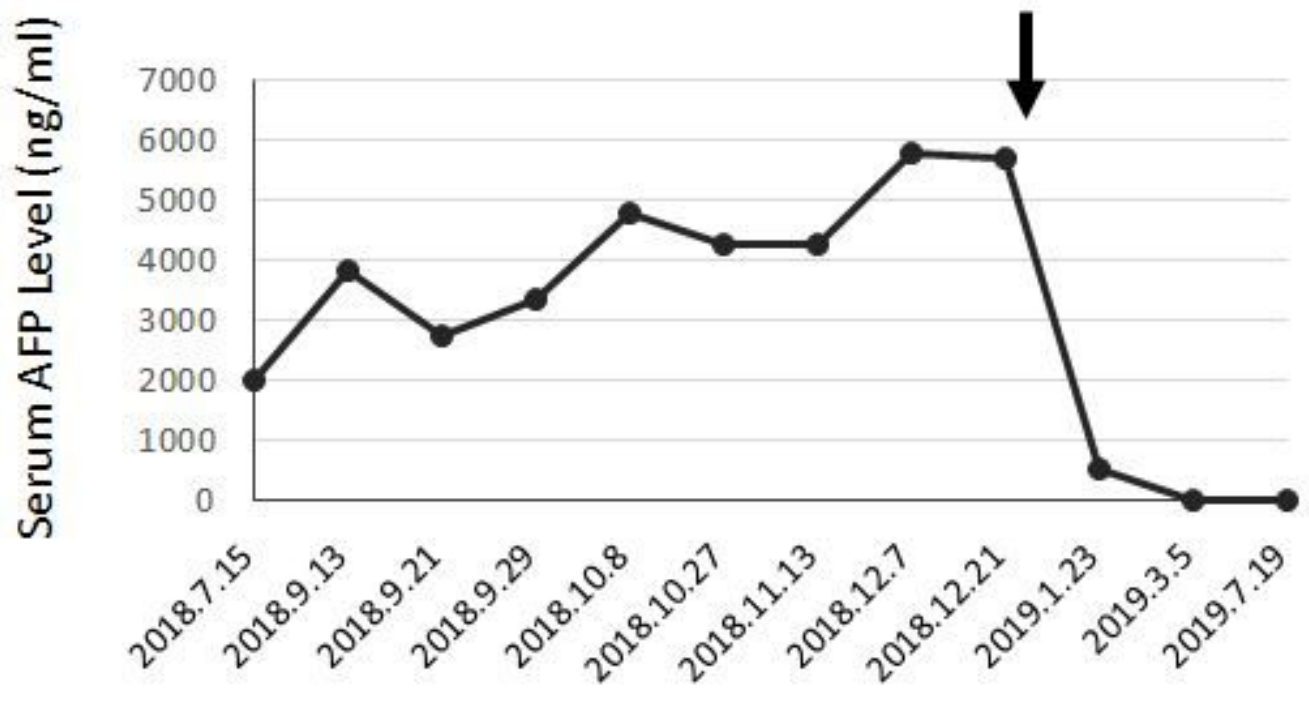

\section{Sampling dates}

\section{Figure 6}

Ketogenic diet reduces serum alpha feta protein (AFP) level. AFP level decreased in response to KD and fell into a normal range. Black arrow indicates the date that the patent began the ketogenic diet. 